\title{
Über kosmische Kernhäufigkeiten \\ II. Mitteilung: Einzelheiten in der Häufigkeitsverteilung der mittelschweren und schweren Kerne
}

\author{
Von Hans E. Suess \\ Aus dem Institut für physikalische Chemie der Universität Hamburg \\ (Z. Naturforschg. 2 a, 604-608 [1947]; eingegangen am 16. Juni 1947)
}

\begin{abstract}
Die in der ersten Mitteilung ${ }^{1}$ durch die Anwendung plausibler Häufigkeitsregeln vorgenommenen Ausgleichungen der G o l d s c h m i d t schen Werte ${ }^{2}$ für die kosmischen Häufigkeiten der Elemente zeigen, daß der durchschnittliche Gehalt der Meteoriten an Se, Te, Ga, In, Tl, Zn, Cd, Hg und Re bedeutend geringer ist als der kosmischen Häufigkeit dieser Elemente entsprechen würde. Die neue Häufigkeitsverteilung läßt die häufigkeitsmäßige Bevorzugung der Kerne mit bestimmten Protonen- und Neutronenzahlen besonders deutlich erkennen. Eine ähnliche, wenn auch weit weniger augenfällige Bevorzugung scheint auch bei bestimmten Neutronenüberschußwerten vorzuliegen.
\end{abstract}

1. Die Goldschmidtschen Häufigkeitswerte

$\mathrm{D}_{\mathrm{i}}^{\mathrm{i}}$ ie älteste geschlossene Zusammenstellung der Häufigkeiten der. Elemente in Meteoriten stammt von I. und W.Nodd a ck ${ }^{3}$. Die Noddackschen Angaben wurden von verschiedenen Autoren, so z. B. von A. E. Fersm a n ${ }^{4}$, kritisch bearbeitet und modifiziert, wobei vor allem das etwas unsicher erscheinende Mengenverhältnis der drei Hauptphasen des meteoritischen Materials, Silicat, Eisen und Sulfid, zur Diskussion stand. Nach einer großen Zahl eigener experimenteller Untersuchungen hat dann Goldschmidt eine vollständige Tabelle sämtlicher Elementhäufigkeiten angegeben, die in manchen Einzelheiten sehr wesentlich von den früheren Angaben der anderen Autoren abweicht. In der ersten Mitteilung ist gezeigt worden, daß aus den Goldschmidtschen Angaben einerseits und der bekannten isotopischen Zusammensetzung der einzelnen Elemente andererseits ein geschlossenes Gesamtbild der kosmischen Häufigkeiten der verschiedenen Kernsorten gewonnen werden kann, wenn man unter Anwendung plausibler Häufigkeitsregeln die Goldschmidtschen Werte für etwa die Hälfte der Elemente in bestimmter Weise modifiziert. Legt man eine der älteren Häufigkeitstabellen zugrunde, dann erhält man im wesentlichen dasselbe Bild der

${ }^{1}$ H. E. Suess, Z. Naturforschg. 2a, 311 [1947].

2 V. M. Gold schmidt, Geochem. Verteilungsges. IX, Videnskapsakademien Oslo 1938.

${ }^{3}$ I. u. W. Nod d a ck, Naturwiss. 8, 757 [1920].
Kernhäufigkeiten, benötigt jedoch höhere Korrekturfaktoren.

Bei den der Modifikation bedürfenden Goldschmidtschen Werten kann man zwei Gruppen von Elementen unterscheiden: eine, für die die Korrekturfaktoren etwa zwischen $1 / 3$ und 3 liegen und dann wohl einer normalen Ungenauigkeitskorrektur entsprechen, und eine zweite, die, offenbar durch eine bedeutende Verarmung des meteoritischen Materials an diesen Elementen, um einen Faktor 5 bis 10 und mehr zu korrigieren ist. Wir wollen diese zweite Gruppe nach Goldschmidt die Gruppe der ,,deficienten Elemente“ nennen.

\section{Die deficienten Elemente}

Die Elemente Se, Te, Ga, In, Tl, Zn, Cd, Hg und Re sind deficiente Elemente und demnach in den Meteoriten erheblich seltener, als man nach den Regeln für die kosmischen Häufigkeiten ihrer Isotope erwarten würde. Es handelt sich hierbei stets um Gruppen von chemisch ähnlichen Elementen, und die Voraussetzung unserer Überlegung, daß diese Verarmung durch sekundäre chemische Einflüsse bedingt ist, erhält damit eine weitere, überzeugende Stütze. Auch der Umstand, daß Unsöld ${ }^{5}$ aus dem Sonnenspektrum für Schwefel und Zink bedeutend höhere Häufigkeitswerte berechnet hat, als Goldschmidt

4 A. E. F e r s m a n, Geochemie, Leningrad 1934.

5 A. Uns öld, Nachr. Ges. Wiss. Göttingen, Math. Physik. Kl., 1945, 69 sowie eine priyate Mitteilung, für die ich Hrn. Prof. Uns öld sehr dankbar bin. 
durch Analyse von Meteoriten erhalten hatte, deutet darauf hin, daß die Goldschmidtschen Werte für diese Elemente und ihre Homologen nicht die wahren kosmischen Häufigkeiten widerspiegeln.

Bei Se und Te tritt (abgesehen von Re) die größte Diskrepanz auf, und zwar ein Faktor 16 bzw. 40. Goldschmidt schreibt über die Elemente der Schwefelgruppe: „Die Häufigkeit dieser drei Elemente im Verhältnis zu anderen Elementen ist schwierig zu erfassen, weil in der Natur in der Regel eine räumliche Trennung der Schwefelverbindungen von anderen geochemischen Phasenkomplexen stattgefunden hat... Über die Häufigkeit des Tellurs liegen erst so wenige Daten vor, daß eine Berechnung des Mengenverhältnisses zu anderen Elementen noch recht unsicher erscheint. Es scheint nur festzustehen, daß Tellur wesentlich seltener als Selen ist, und daß das Gewichtsverhältnis zwischen beiden Elementen vielleicht von der Größenordnung 1: 50 ist.“ Für Schwefel ergab die Berechnung des Häufigkeitswertes auf der Sonne von Unsöld einen um einen Faktor 3,3 höheren Wert; die Interpolation für $\mathrm{S}^{33}$ scheint einen noch etwas höheren Wert nahezulegen. Somit scheinen die Meteoriten auch an Schwefel verarmt zu sein.

Als wesentlich genauer sind die Golds chmidt . schen Werte für Ga, In und $\mathrm{Tl}$ zu betrachten. Auch bei der Interpolation lassen sich die Isotopenhäufigkeiten in den Linienzug für die ungeraden Massenzahlen gut einpassen und die gewählten Werte für die Korrekturfaktoren (Ga: 8,0, In: 3,2 und Tl: 5,2) dürfen relativ $\mathrm{zu}$ den Nachbarelementen innerhalb von $20 \%$ als genau gelten.

Für $\mathrm{Zn}, \mathrm{Cd}$ und $\mathrm{Hg}$ bezeichnet Goldschmidt die Daten als noch nicht sehr befriedigend. Uber die Menge des $\mathrm{Cd}$ und $\mathrm{Hg}$ in Meteoriten liegen nur ganz wenige Daten vor. Die Ausgleichung läßt sich für diese Elemente besonders gut durchführen und die korrigierten Werte dürften relativ zu denen ihrer Nachbarn kaum einen. Fehler von mehr als 10\% aufweisen. Die Korrekturfaktoren sind: Zn: 4,2, Cd: 1,9 und $\mathrm{Hg}$ : 6,3. Für Zink gibt Unsöld einen um den Faktor 6 höheren Wert an als Goldschmidt; der interpolierte Wert liegt also zwischen diesen Angaben.

Einigermaßen rätselhaft erscheint die einzigartig abnorme Seltenheit des Re, wie sie von I. und W. $\mathrm{Nodd} \mathrm{a} \mathrm{ck} \mathrm{k}^{6}$ bei der Analyse von Meteoriten festgestellt wurde. Das Häufigkeitsverhältnis der beiden Isotopen dieses Elements paßt, obwohl an einer „Sprungstelle“ liegend, so gut in den weiteren Verlauf der Häufigkeitslinie, daß für die Absolutwerte auch ein Korrekturfaktor von über 2 Zehnerpotenzen gerechtfertigt erscheint.

Zur Erklärung der Verarmung der Meteoriten an diesen Elementen könnte man annehmẹn, daß diese Elemente oder eine ihrer Verbindungen bei

B I. u. W. Noddack, Z. physik. Chem., Abt. A, 154, 201 [1931]. entsprechend hoher Temperatur so flüchtig sind, daß sie bei der Bildung der Meteoriten nur unvollkommen kondensiert wurden. Diese Vorstellung erscheint jedoch aus verschiedenen Gründen wenig befriedigend. Ein Hinweis auf eine weitere Deutungsmöglichkeit des Befundes ergibt sich aus dem Umstand, daß sämtliche genannten Elemente (mit 'Ausnahme des Re) nach Noddack vorzugsweise in der Sulfidphase, dem Troilit, enthalten sind. Freilich schreibt Goldschmidt: „Der Stoffbestand, den I. und W. Noddack als Durchschnittsanalyse der Troilitknollen aus fünf großen Eisenmeteoriten angegeben haben, ist jedenfalls weder der gewöhnliche Stoffbestand von Troilit von Eisenmeteoriten, noch von Troilit der Silikatmeteoriten. Nach unserer heutigen Kenntnis kann man vielmehr sagen, daß nur einige wenige Elemente sich in den Meteoriten chalkophil verhalten, also vorzugsweise in Troilitknollen eingehen; dies sind vor allem Schwefel, Selen, Chrom, Silber und Zink; von einigen anderen (wie z.B. Arsen und Antimon) erscheint die Frage noch ungeklärt." Nun ist - in Zusammenhang mit der Frage der Herkunft der Tektite - von $\mathrm{Mich}$ e $\mathrm{l}^{7}$ die Vermutung ausgesprochen worden, daß eine ganze Klasse von Meteoriten der Erfassung entgeht, nämlich die der vorzugsweise aus Oldhamit (Schwefelcalcium) und anderen Leichtmetallsulfiden bestehenden, und zwar, -weil sie bei ihrem Sturz auf die Erde in der Atmosphäre verbrennen. Mag auch ihre Gesamtmenge gering sein, so ist es doch denkbar, daß sie die fehlenden Hauptmengen der „deficienten“" Elemente enthalten; denn es ist wohl anzunehmen, daß in den Leichtmetallsulfiden zwär nicht genau die gleichen, aber doch ungefähr dieselben Elemente an gereichert sind wie in der Eisensulfidphase.

\section{Die Häufigkeitswerteder übri- gen Elemente}

Die in Tab. 1 angegebenen interpolierten Werte, die mit den Angaben und Abbildungen der I. Mitteilung übereinstimmen, wurden bei einer ersten und vorläufigen Ausgleichung gewonnen. Bei einer neuerlichen Überarbeitung, die noch beabsichtigt ist, wird sich vermutlich zeigen, daß an einigen Stellen einem etwas anderen Verlauf der Häufigkeitslinien der Vorzug zu geben ist. Hier-

7 H. M i c h e l, Fortschr. Mineral. Kristallogr. Petrogr. 23, 1 [1939]. 


\begin{tabular}{|c|c|c|c|c|c|c|c|c|c|}
\hline$Z$ & $\begin{array}{l}\text { Ele- } \\
\text { ment }\end{array}$ & $\begin{array}{c}\text { Nach } \\
\text { Unsöld }\end{array}$ & $\begin{array}{l}\text { Nach } \\
\text { Goldschmidt }\end{array}$ & $\begin{array}{c}\text { vorläufiger } \\
\text { interpolierter } \\
\text { lV ert }\end{array}$ & $Z$ & $\begin{array}{l}\text { Ele- } \\
\text { ment }\end{array}$ & $\begin{array}{c}\text { Nach } \\
\text { Unsöld }\end{array}$ & $\begin{array}{c}\text { Nach } \\
\text { Goldschmidt }\end{array}$ & $\begin{array}{c}\text { vorläufiger } \\
\text { interpolierter } \\
\text { Wert }\end{array}$ \\
\hline 1 & $\mathrm{H}$ & 4,3 & - & $\longrightarrow$ & 43 & $\mathrm{Tc}$ & - & - & - \\
\hline 2 & $\mathrm{He}$ & 3,5 & - & - & 44 & $\mathrm{R}_{11}$ & - & $-5,44$ & $-5,44$ \\
\hline 3 & $\mathrm{Li}$ & - & $-4,0$ & - & 45 & $\mathrm{Rh}$ & - & $-5,86$ & $-6,02$ \\
\hline 4 & $\mathrm{Be}$ & - & $-4,72$ & - & 46 & $\mathrm{Pd}$ & - & $-5,60$ & $-5,47$ \\
\hline 5 & B & - & $-4,61$ & - & 47 & Ag & - & - 5.59 & $-5,79$ \\
\hline 6 & C & $(0,94$ & - & - & 48 & $\mathrm{Cd}$ & - & $-5,59$ & $-5,32$ \\
\hline 7 & $\mathrm{~N}$ & 1,16 & - & - & 49 & In & - & $-6,64$ & $-6,15$ \\
\hline 8 & 0 & 1,28 & - & - & 50 & Sn & $\ldots$ & $-4,54$ & $-5,(1) 4$ \\
\hline 9 & $\mathrm{~F}$ & - & $-2,82$ & - & 51 & $\mathrm{Sb}$ & - & -6.14 & $-6,14$ \\
\hline 10 & $\mathrm{Ne}$ & 1.36 & $=-$ & - & 52 & $\mathrm{Te}$ & - & $-6,70$ & $-5,10$ \\
\hline 11 & $\mathrm{Na}$ & $-1,17$ & $-1,36$ & - & 53 & $\mathbf{J}$ & - & $-5,87$ & $-5,97$ \\
\hline 12 & $\mathrm{Mg}$ & 0,06 & $-11,06$ & - & 54 & $\mathrm{X}$ & - & - & -5.43 \\
\hline 13 & Al & $-1,12$ & $-1,06$ & - & 55 & Cs & - & $-7,00$ & $-6,45$ \\
\hline 14 & $\mathrm{Si}$ & $\mathbf{u},-$ & (1),- & $0, \cdots$ & 56 & $\mathrm{Ba}$ & $-4,4$ & $-5,11$ & $-5,11$ \\
\hline 15 & $\mathrm{P}$ & - & $-2,23$ & - & 57 & La & - & $-\mathbf{5 , 6 8}$ & $-\mathbf{5 , 6 8}$ \\
\hline 16 & $\mathrm{~S}$ & $-0,53$ & $-0,44$ & - & 58 & $\mathrm{Ce}$ & - & $-5,28$ & -5.28 \\
\hline 17 & $\mathrm{Cl}$ & - & $-2,41$ & - & 59 & $\mathrm{Pr}$ & - & $-6,02$ & $-6,02$ \\
\hline 18 & A & - & - & $(-0,9)$ & 60 & $\mathrm{Nd}$ & - & $-5,48$ & $-5,48$ \\
\hline 19 & $\mathbf{K}$ & $-2,35$ & $-2,16$ & - & 61 & & - & - & - \\
\hline 20 & $\mathrm{Ca}$ & $-1,22$ & $-1,24$ & - & 62 & $\mathrm{Sm}$ & - & $-5,94$ & $-5,94$ \\
\hline 21 & Sc & -4.12 & $-4,82$ & - & 63 & Eu & - & - 6.ร๊5 & $-6,55$ \\
\hline 22 & $\mathrm{Ti}$ & $-2,49$ & $\ldots 2,33$ & - & 64 & Gd & - & $-5,78$ & -5.78 \\
\hline 23 & V & $-3,40$ & $-3, \diamond 9$ & - & 65 & Tb & - & $-6, \geq 8$ & $-6,28$ \\
\hline 24 & $\mathrm{Cr}$ & $-1,87$ & $-1,45$ & - & 66 & Dy & - & $-5,69$ & $-5,69$ \\
\hline 25 & Mn & $-1,99$ & $-2,18$ & 一 & 67 & Ho & - & $-6,24$ & $-6,2 t$ \\
\hline 26 & $\mathrm{Fe}$ & 0,27 & $(1,1) 6$ & - & 68 & $\mathrm{Er}$ & - & $-5,79$ & $-5,79$ \\
\hline 27 & Co & $-2,42$ & $-2.4 h^{\circ}$ & - & 69 & $\mathrm{Tm}$ & - & $-\mathbf{f , 5 4}$ & $-6,5 t$ \\
\hline 28 & $\mathrm{Ni}$ & -1.50 & $-1,34$ & - & 70 & $\mathrm{Yb}$ & - & $-5,82$ & $-5,82$ \\
\hline 29 & $\mathrm{Cu}$ & -3.22 & $-3,34$ & $-3,+i 4$ & 71 & $\mathrm{Cp}$ & 一 & $-t ;, 31$ & $-6,31$ \\
\hline 30 & $\mathrm{Zn}$ & $-2,67$ & $-3,+4$ & $-2, \wedge 2$ & 72 & $\mathrm{Hf}$ & - & $-5,82$ & $-5,52$ \\
\hline 31 & $\mathrm{Ga}$ & - & $-5,(18$ & $-4,18$ & 73 & $\mathrm{Ta}$ & - & $-5,55$ & $-6,35$ \\
\hline 32 & $\mathrm{Ge}$ & - & $-3,73$ & $-3,49$ & 74 & W & - & $-4, \times 4$ & $-5,34$ \\
\hline 33 & As 8 & - & $-4,74$ & $-4,74$ & 75 & $\operatorname{Re}$ & 一 & $-8,75$ & $-6,65$ \\
\hline 34 & $\mathrm{Se}$ & - & $-4,83$ & -3.63 & 76 & Os & - & $-5,76$ & -5.76 \\
\hline 35 & $\mathrm{Br}$ & - & $-4,37$ & $-4,37$ & 77 & $\mathrm{Ir}$ & 一 & $-6, \geq 4$ & $-5,999$ \\
\hline 36 & $\mathrm{Kr}$ & - & - & $-3,72$ & 78 & $\mathrm{Pt}$ & - & $-5,54$ & -5.54 \\
\hline 37 & $\mathrm{Rb}$ & - & $-5,17$ & $-4,62$ & 79 & $\mathrm{Au}$ & - & $-6, \underline{4}$ & $-6.2 t$ \\
\hline 38 & $\mathrm{Sr}$ & $-4,00$ & $-4,44$ & -4.24 & 80 & $\mathrm{Hg}$ & - & $-\hbar, 52$ & $-5,72$ \\
\hline 39 & $Y$ & $-4,14$ & -5.111 & $-5,01$ & 81 & $\mathrm{TI}$ & - & $-6,77$ & $-5,97$ \\
\hline 40 & $\mathrm{Zr}$ & $-4,98$ & $-3,86$ & -4.24 & 82 & $\mathrm{~Pb}$ & $-4,8$ & $-5,113$ & - \\
\hline 41 & $\mathrm{Nb}$ & - & -5.16 & $-5,41$ & 83 & $\mathrm{Bi}$ & - & $-6,94$ & - \\
\hline 42 & Mo & $-5,57$ & $-5,(12$ & $-5,02$ & & & & & \\
\hline
\end{tabular}

Tab. 1. Dekadische Logarithmen der Elementhäufigkeiten, bezogen auf $H_{\mathrm{Si}}=1$.

bei werden zwar die interpolierten Häufigkeitswerte mancher Elemente noch eine Änderung bis zu etwa einem Faktor 2 erfahren können; relativ zu den Werten der Nachbarelemente jedoch werden im allgemeinen keine merklichen Änderungen eintreten.

Für die folgenden Elemente wurden vorerst die Gol d s c h m i d schen Werte unverändert übernommen: Seltene Erden sowie Y, As, Sb, Bi, Br, Mo, Ba, $\mathrm{Ru}, \mathrm{Os}, \mathrm{Pt}$ und $\mathrm{Au}$. Der Häufigkeitswert für Sn ist um den Faktor 0,32 herabgesetzt worden; man kann jedoch den Goldschmidtschen Wert beibehalten und statt dessen die Werte für In, Cd, Pd und $\mathrm{Ru}$ weiter hinaufsetzen. Die Werte für Te und $\mathrm{X}$ stützen sich auf die Annahme, daß der Goldschmidtsche Wert für Sb beibehalten werden kann. Auffallend ist, daß es zweifellos notwendig ist, auch bei chemisch so ähnlichen Elementen wie der Gruppe der Platinmetalle: $\mathrm{Ru}, \mathrm{Rh}, \mathrm{Pd}, \mathrm{Os}, \mathrm{Ir}$ und $\mathrm{Pt}$ individuelle Korrekturen anzubringen. Ebenso scheint es sicher, daß der Goldschmidtsche Wert für $\mathrm{Zr}$ auf weniger als die Hälfte vermindert werden muß. Als besonders unsicher muß die Interpolation der Werte für $\mathrm{Hf}$, Ta und $\mathrm{W}$ gelten ${ }^{8}$. Für die Elemente $\mathrm{Rb}$ und $\mathrm{Cs}$ wurde ein Kor

$8 \mathrm{Hr}$. Prof. Goldsch midt hatte die Freund lichkeit, mir in einem Briefe vom 9. Januar 1947 mitzuteilen, daß sein Wert für Tantal nach $\mathrm{R}$ a $\mathbf{n}$ $\mathrm{k} \mathrm{a} \mathrm{m}$ a auf etwa $1 / \mathrm{g}$ zu vermindern ist. Danach stim men nunmehr der empirische und der interpolierte Wert für Ta fast vollkommen überein. 


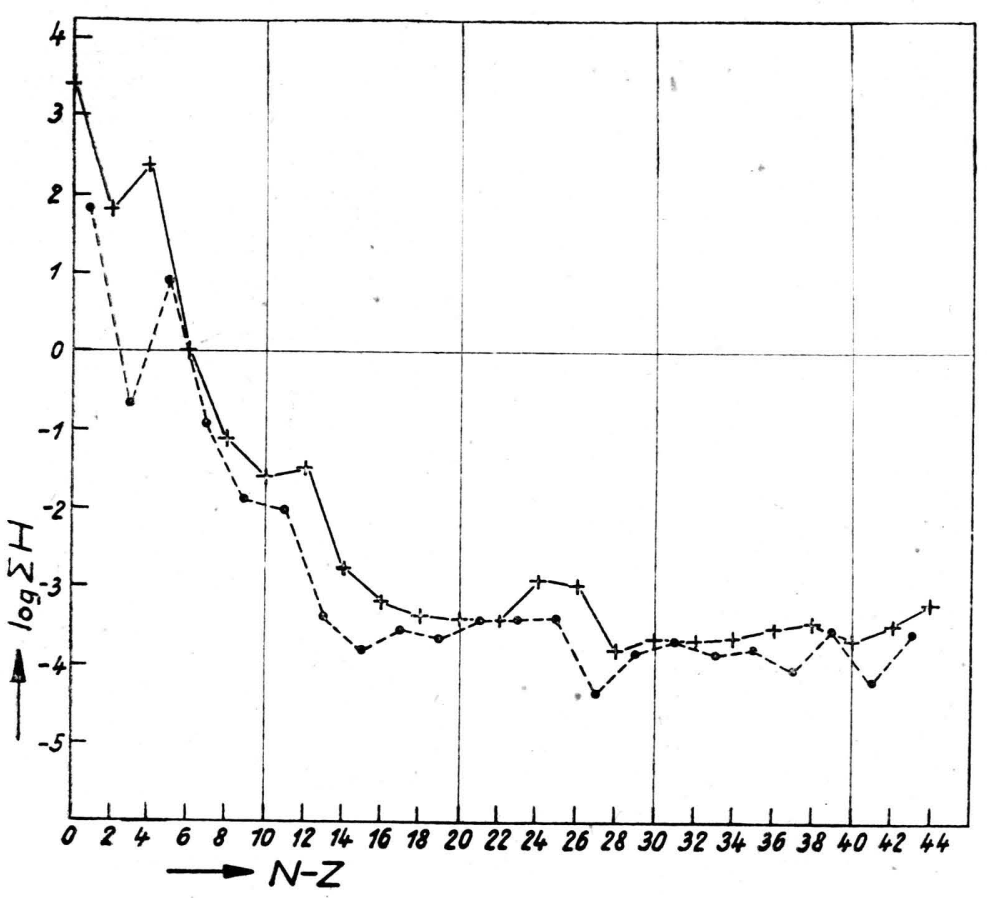

Abb. 1. Abszisse: Neutronenüberschuls $N-Z$. Ordinate: Dekadische Logarithmen der Summen der Häufigkeiten aller Kernc mit gleichem Neutronenüherschuf.

rekturfaktor 3,5 gewählt, der jedoch für Ce um einen Faktor 2 unsicher ist. Bei Rb liegen besonders interessante Verhältnisse vor. Das mit dem $\mathrm{Sr}^{87}$ isobare Rb-Isotop ist bekanntlich $\beta^{-}$-aktiv. Die Summe der Häufigkeiten der beiden Isobaren sollte auf der Häufigkeitslinie für die Kerne mit ungerader Massenzahl liegen; das ist jedoch nur annähernd, aber nicht genau der Fall. Diese Abweichung ist aber ohne weitereverständlich, wenn man annimmt, daß das „Alter“ der Probe, von der die isotopische Zusammensetzung des Sr bestimmt wurde, d. h. die Zeit, die vergangen ist seit der Abtrennung des Sr der Probe von der der kosmischen Häufigkeitsverteilung entsprechenden Menge von Rb, nicht sehr klein ist gegen die Halbwertszeit ${ }^{9}$ des $\mathrm{Rb}^{87}$.

In die Tab. 1 ist auch der ungefähre Wert für A aufgenommen, obwohl die Interpolation bei den leichteren Elementen wegen der stärkeren Schwankungen in den Häufigkeitslinien schwieriger und weniger eindeutig ist. Hierbei ist die Häufigkeit des A40 nicht mitberücksichtigt, dessen irdische Häufigkeit nicht der kosmischen entspricht. Die Werte für $\mathrm{Kr}$ und $\mathrm{X}$ können die gleiche Genauigkeit beanspruchen wie die der übrigen Elemente.

9 Aus den angegebenen Häufigkeitswerten für Rb und $\mathrm{Sr}$ ergibt sich ein sicherlich zu hohes Probenalter, jedoch würde eine weitere, geringfügige Korrektur eines der beiden Werte bereits einen vernünftigen Wert ergeben.
Abgesehen von denen der deficienten Elemente, liegen die Ausgleichsfaktoren wohl im wesentlichen innerhalb der Genauigkeitsgrenzen der Goldschmidtschen Häufigkeitsangaben, und es ist erstaunlich, daß es möglich ist, bereits mit so kleinen Korrekturen zu einem geschlossenen Gesamtbild der Häufigkeitsverteilung zu gelangen, wenn man bedenkt, welche weit voneinander abweichenden Analysenergebnisse gosichtet und wie gewagt mitunter die notwendigen Mittelwertbildungen vou Goldschmidt vorgenommen werden mußten.

1. Andeutungen für kernphysikalische Zusammenhänge

Von allerf experimentellen Daten, die die Grundlage einer Theorie des Baues der schwereren Kerne bilden könnten, sind die wichtigsten, die der Bindungsenergie und Anregungsspektren, nur sehr unvollständig bekannt. Es erscheint daher naheliegend, bei den Daten für die kosmischen Häufigkeiten der stabilen Kerne, die sicherlich, wenn auch vielleicht in sehr verwickelter Weise. mit ihren Bindungsenergien in Zusammenhang stehen ${ }^{10}$, nach Hinweisen zu suchen, die uns einem Verständnis ihres Aufbaues näher bringen könnten. In Abb. 7 der ersten Mitteilung ist die bekannte Tatsache sehr klar zu erkennen, dal Kerne bestimmter Neutronen- und Protonenzahl häufigkeitsmäßig bevorzugt sind. Auf das Vorliegen solcher ausgezeichneten Zahlen, die man bereits ohne Häufigkeitsbetrachtung auf Grund der Anzahl der stabilen Isotope erkennen kann, hat bereits Elsa sse ${ }^{11}$ aufmerksam gemacht, und zwar für $N$ und $Z=50$ und 82 . Unabhängig davon hat Goldschmidt² auf die auffallend hohe kos-

10 Vgl. H. Jensen u. H. Su ess, Naturwiss, in Druck.

11 W. Elsasser', J. Physique Radium 4, 549 [1933]; 5, 389, 636 [1934].

12 Hrn. Hax e l, Göttingen, danke ich für interessante Diskussionen über diese ausgezeichneten Zahlenwerte und insbesondere für seinen Hinweis auf die Zahl 28, die für $N$ in der Tat sehr deutlich hervortritt. Für $Z$ erscheint häufigkeitsmäßig die Zahl 26 bevorzugt; die in diesem Gebiet einigermaßen bekannten Bindungsenergien weisen jedoch auf eine Bevorzugung der Zahl 28; der Unterschied wäre als 
mische Häufigkeit dieser Kerne hingewiesen. Diese Bevorzugung liegt bei den folgenden Werten vor ${ }^{12}$ :

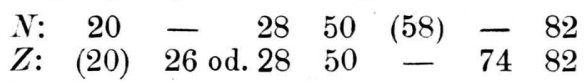

Die éingeklammerten Zahlen geben Werte an, bei denen die Bevorzugung nur wenig hervortritt und bei denen eine kernphysikalische Bedeutung noch ungewiß erscheint.

Die Abb. 7 und 8 lassen jedoch auch eine sprunghafte Häufigkeitsverminderung in gewissen „Zwischengebieten“erkennen, und zwar vor allem bei den Kernen, für die $Z>50$ und $N<82$ ist, sowie für die Kerne, deren $Z$ zwischen 74 und 82 liegt. Eine ähnliche Häufigkeitsverminderung scheint auch vorzuliegen bei den Kernen mit $Z>20$ und $N<28$.

eine Folge einer komplizierteren Entstehungskinetik dieser Kerne aufzufassen.

Anm. bei der Korrektur: Ein weiterer interessanter Beleg für die energetische Bevorzugung von Kernen dieser Neutronenzahlen ist dem Bericht des Plutonium Project, J. amer. chem. Soc. 68, 2411 [1946], der nach Fertigstellung des Manuskripts durch den Auszug von J. M a t t a u ch, diese Z. 2a, 120 [1947], hier bekannt geworden ist, zu entnehmen. Danach sind die bislang einzigen als Neutronenstrahler identifizierten Kerne das $\mathrm{Kr}^{87}$ und das $\mathrm{X}^{137}$. Ihre Neutronenzahl beträgt $51 \mathrm{bzw}$. 83; durch Emission eines Neutrons gehen sie in einen ,bevorzugten“ Kern mit der Neutronenzahl 50 bzw. 82 über.
Während diese Befunde als gesichert gelten können, läßt sich aus dem Bilde, das die Häufigkeitsverteilung bietet, auch eine Reihe weiterer Periodizitäten und Regelmäßigkeiten herauslesen, von denen nicht gesagt werden kann, ob sie zufällig oder durch kernphysikalische Verhältnisse bedingt sind. Es sei hier nur noch erwähnt, daß auch Kerne mit bestimmten Neutronenüberschußwerten eine häufigkeitsmäßige Bevorzugung aufzuweisen scheinen. In der Abb. 1 sind die Häufigkeitssummen der Kerne mit gleichem Neutronenüberschuß aufgetragen. Die Linie, die die Werte der Häufigkeitssummen für Kerne mit geradem Neutronenüberschuß (Kreuze, im wesentlichen $g-g$-Kerne) verbindet, hat einen auffallend glatten Verlauf, wenn man von den Punkten für die folgenden Werte absieht, bei denen eine „häufigkeitsmäßige Bevorzugung" vorzuliegen scheint:

$$
N-Z: \quad 4, \quad 12, \quad 24, \quad 26, \quad(38) .
$$

Die entsprechende Linie für die Kerne mit ungeradzahligem Neutronenüberschuß (gestrichelt, $g-u$ - und $u-g$-Kerne) verläuft weniger glatt; hier ist jedoch an Stellen, wo stabile oder fast stabile Isobarenpaare vorliegen, die Häufigkeitszuordnung noch unsicher oder der Linienverlauf als vom Weltalter abhängig zu betrachten.

\title{
Geometrie und Kristallphysik einer Röntgenlinse für monochromatische Strahlung
}

\author{
Von Walter Hoppe* und Hans Jochen Trurnit \\ (Z. Naturforschg. 2 a, 608 615 [1947]; eingegangen am 7. Juli 1947)
}

\begin{abstract}
Es wird die Geometrie und Kristallphysik einer Linse für monochromatische Röntgenstrahlung behandelt, die durch Uberziehen von Rotationsformkörpern mit Kristallschichten entsteht. Von den durch Biegung von Kristallen erhaltenen Röntgenabbildungssystemen unterscheidet sich die neue Linse vor allem durch Formung der abbildenden Netzebenen nach nichtabrollbaren Flächen. Dadurch erhält sie sehr gute geometrisch-optische Abbildungseigenschaften, rotationssymmetrischen Strahlengang für Achsenpunkte mit Gültigkeit der Linsengesetze und kann daher zur bequemen Einstellung mit Lichtlinsen gleicher Brennweite kombiniert werden. Sie hat aber wie alle Kristallinsen einen starken chromatischen Fehler (monochromatische Strahlung erforderlich) und eine ,flächenhafte“ Apertur, daher relativ geringe Lichtstärke. Es wird ein Weg zur Erreichung einer ,räumlichen“ Apertur angegeben. Eine maximale Auflösung von etwa $1 \mu$ erscheint erreichbar.
\end{abstract}

\section{Alloemeines}

$\mathrm{R}$ öntgenstrahlung hat in allen Medien einen Brechungsexponenten von nahezu 1; aus diesem Grunde sind Röntgenlinsen und -spiegel nicht möglich und die optischen Anordnungen in der
Röntgenographie arbeiten mit Schattenwurf und Blenden als optischen Hilfsmitteln.

Eine Röntgenoptik hätte Interesse für die $\mathbf{A b}$ bildung von Gegenständen mit Röntgenstrahlen

* München-Solln, Memlingstr. 4. 